\title{
Direct Carotid Cavernous Fistulas
}

Satomi Ide, ${ }^{1}$ Hiro Kiyosue, ${ }^{1}$ Kohei Tokuyama, ${ }^{1}$ Yuzo Hori, ${ }^{2}$ Yoshiko Sagara, ${ }^{3}$ and Takeshi Kubo ${ }^{4}$

A direct carotid cavernous fistula (CCF) is an abnormal shunt between the internal carotid artery (ICA) and the cavernous sinus (CS). Traumatic CCF is the most common type, accounting for up to $75 \%$ of all CCFs. For the management of direct CCF, endovascular therapy has become the standard.

For successful endovascular therapy, evaluation of the size and location of orifice of the CCF, venous drainage, and tolerance for ICA occlusion on cerebral angiography is necessary. Multi-planner reformatted images of 3D rotation angiography are useful to visualize the fistula and compartments of the CS precisely.

Due to the limited commercial availability of detachable balloons, detachable coils have become a widely employed endovascular tool for the treatment of direct CCFs. The advantageous aspects of coil application are their easy retrievability and better control. In the case of large/multiple fistulas, adjunctive techniques, including balloon- and stent-assisted techniques, are often needed to occlude the CCF while preserving the ICA. To avoid cranial nerve palsy related to over-packing of the CS with detachable coils or a detachable balloon, selective embolization of the fistula portion is required. Use of liquid embolic materials and covered stents was recently reported as another adjunctive technique. In cases in which it is impossible to occlude the CCF while preserving the ICA, parent artery occlusion (PAO) is considered. The selection of additional/alternative techniques and devices depends on the anatomy and hemodynamics of each CCF, and the skill and experience of individual operators.

Keywords $>$ direct carotid cavernous fistula, transarterial embolization, transvenous embolization, coil embolization, detachable balloon

\section{Introduction}

A direct carotid cavernous fistula (CCF) is an abnormal arteriovenous shunt between the internal carotid artery (ICA) and the cavernous sinus (CS). Etiological classification separates CCFs into traumatic CCF and spontaneous CCF.

${ }^{1}$ Department of Radiology, Oita University Faculty of Medicine, Yufu, Oita, Japan

${ }^{2}$ Department of Radiology, Nagatomi Neurosurgical Hospital, Oita, Oita, Japan

${ }^{3}$ Department of Radiology, Tsurumi Hospital, Beppu, Oita, Japan ${ }^{4}$ Department of Neurosurgery, Oita University Faculty of Medicine, Yufu, Oita, Japan

Received: July 2, 2020; Accepted: September 1, 2020

Corresponding author: Satomi Ide. Department of Radiology, Oita University Faculty of Medicine, 1-1, Idaigaoka, Hasamamachi, Yufu, Oita 879-5593, Japan

Email: idesat@oita-u.ac.jp

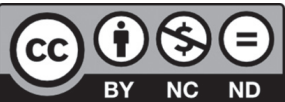

This work is licensed under a Creative Commons Attribution-NonCommercialNoDerivatives International License.

(C)2020 The Japanese Society for Neuroendovascular Therapy
Traumatic CCF is the most common type, accounting for up to $75 \%$ of all CCFs. ${ }^{1,2}$ The shunt flow in direct CCFs is generally markedly high and causes numerous symptoms, including exophthalmos, diplopia, epistaxis, and cerebral hemorrhage. Emergency treatment is often required in cases with severe symptoms and high-risk venous drainage.

Cases of direct CCF used to be mainly treated by muscle occlusion, carotid artery ligation, or a combination of these methods. Following the advent of useful devices, such as detachable balloons and coils, endovascular therapy has become the standard management for direct CCFs.

To evaluate the size and location of a fistula, and tolerance for ICA occlusion, cerebral angiography is an important examination. The treatment method depends on angiographic findings. This review presents important issues for the treatment of direct CCFs.

\section{Classification and Etiology}

Barrow et al. ${ }^{3)}$ classified CCFs into four types based on arterial supply. Type A is a direct fistula between the ICA and 
the CS. Type A fistulas usually present with a high-flow shunt. Type B fistulas have dural branches of the ICA to the $\mathrm{CS}$, which are comparatively uncommon. Type C fistulas have dural branches of the external carotid artery (ECA) to the CS. Type D fistulas have dural branches of both the ICA and ECA to the CS. ${ }^{3)}$ Type A CCFs are usually caused by head trauma, termed traumatic direct CCFs. Approximately $20 \%$ of type A CCFs are caused by the rupture of either a cavernous segment aneurysm or a weakened atherosclerotic artery. ${ }^{4)}$ Predisposing factors associated with the development of spontaneous type A CCFs are Ehlaers-Danlos syndrome, fibromuscular dysplasia, and pseudoxanthoma elasticum. ${ }^{3)}$ Special attention should be paid particularly for Ehlaers-Danlos syndrome because many vascular events, including CCF, arterial dissection, and pseudoaneurysm, can subsequently develop. ${ }^{5)}$

Iatrogenic damage (trans-sphenoidal surgery and glycerol rhizotomy) may lead to direct CCFs. Recently, direct CCFs were reported as a complication of neuroendovascular treatment, including flow diverter treatment for cavernous ICA aneurysms. Roy et al. ${ }^{6}$ ) found CCFs in $11.4 \%$ of 44 patients during the pipeline endovascular device (PED) procedure. Lin et al. presented two cases of direct CCFs that formed as a result of delayed aneurysm rupture following PED treatment for cavernous ICA aneurysms. ${ }^{7)}$ Matsumoto et al. reported a case of direct CCF during withdrawal of a stent retriever device for the treatment of acute middle cerebral artery occlusion. ${ }^{8)}$

Regarding the location of fistulas, the most frequent location of direct CCF was reported to be the proximal horizontal intracavernous segment of the ICA, followed by the junction of the horizontal and ascending segments.9)

\section{Clinical Features}

The majority of patients with direct CCF present with proptosis $(90 \%)$ and chemosis $(90 \%)$. Other common symptoms include diplopia (50\%), trigeminal nerve dysfunction, and increased intraocular pressure. Visual loss also develops in some patients. Intracranial hemorrhage develops in $5 \%$ of patients due to venous hypertension from cortical venous reflux. Life-threatening epistaxis caused by rupture of a varix in the drainage route through the nasopharynx develops in $1 \%-2 \%{ }^{4}$ )

Cranial nerve palsies may be a symptom of CCFs. The most commonly affected cranial nerves are the $3 \mathrm{rd}$ and 6 th nerves. Bruit due to direct CCF is observed in $25 \%$ of patients. Therefore, if ptosis and 6th nerve palsy or bruit after trauma is noted, careful clinical examination to detect eye bruit, or Doppler US, CT, or MRA to assess for direct $\mathrm{CCF}$ is needed (Fig. $\mathbf{1 a}$ and $\mathbf{1 b}) .{ }^{10)}$

\section{Radiological Evaluation}

Cerebral angiography is the gold standard for the diagnosis, evaluation of hemodynamics, and planning of endovascular intervention for direct CCFs. Evaluation items on angiography include the location and size of the fistula, venous drainage, and cerebral arterio-venous circulation.

In the evaluation of direct CCFs, identifying the exact location of fistulous communication in the cavernous ICA can be challenging because contrast media can be rapidly washed out without sufficient opacification at the fistula and CS due to high shunt flow.

High-resolution DSA with a high frame rate $(>5$ frames/s) and rapid contrast injection rates is required for evaluating the morphology of high-flow fistulas. ${ }^{11)}$ However, high-flow CCFs may be difficult to capture on angiography even with high frame rates. Specific maneuvers can be implemented to slow the fistula flow and facilitate image capture.

Temporal occlusion of the ipsilateral carotid artery by manual compression or balloon occlusion during injection into the contralateral ICA and vertebral artery (Matas and Allcock maneuvers) (Fig. 1c-1g) makes the fistula opaque through the anterior and posterior communicating arteries. Considering the risk of radiation exposure to the practitioner and the effects of motion artifact, balloon occlusion is preferable to manual compression. The tolerance for ICA occlusion should be evaluated using the balloon occlusion test (BOT) to aid in the identification of appropriate therapeutic choices. Reconstruction of 3D rotation angiography images is a useful tool to detect fistulas between the ICA and CS. To evaluate the size and location of the ICA, axial and coronal multiplanar reconstruction (MPR) reformatted images from rotation angiography can more precisely visualize the fistula (Figs. 1 and 2).

\section{Endovascular Treatment}

Since the first treatment of CCF by Travers et al. by ligature of the common carotid artery in the neck in $1809,{ }^{12}$ ) direct CCFs were mainly treated by muscle occlusion, carotid artery ligation, or a combination of these methods.

In 1969, Serbinenko treated a CCF using a non-detachable silicone balloon and then using a detachable balloon in 

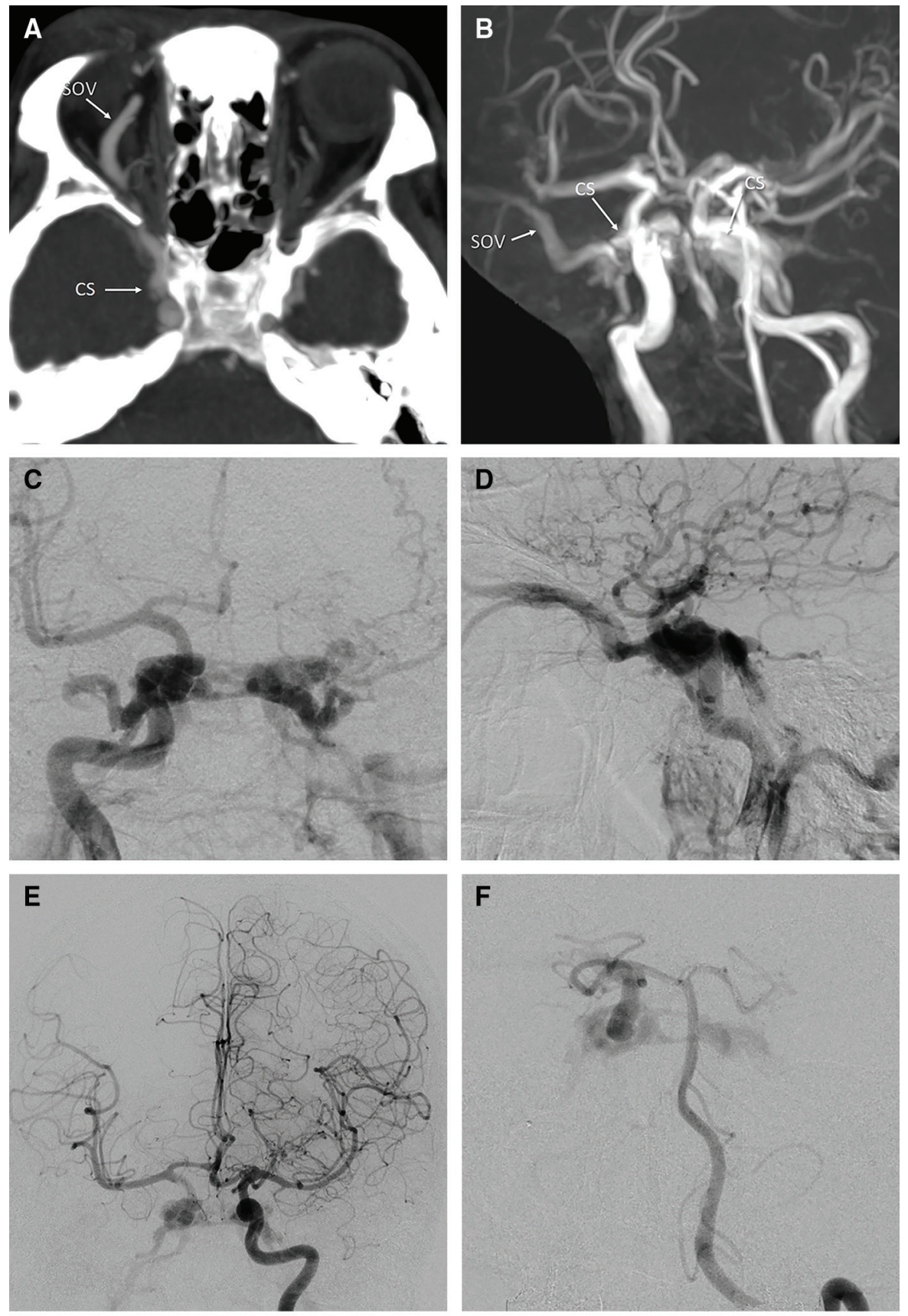

Fig. 1 A 43-year-old male presented with proptosis, chemosis, and diplopia after a traffic accident. (A) Axial partial MIP enhanced CT image showing dilatation of the right SOV and the right CS. (B)

Right anterior oblique view on 3D-TOF MRA showing the dilated right SOV and the bilateral CSs. (C, D) Frontal (C) and lateral (D) views on right internal carotid angiography showing a direct high-flow shunt between the horizontal intracavernous segment of the right ICA and the CS. The direct CCF drained into the contralateral CS via the inter-CS, the bilateral SOVs, and the bilateral inferior petrosal sinuses. The left superficial middle cerebral vein and the superior petrosal sinus drained retrogradely into the bilateral superior ophthalmic vein and bilateral inferior petrosal sinus. On the left side, dangerous cerebral venous refluxes, including the superficial middle cerebral vein, uncal vein, and the petrosal vein were observed. (E) Left internal carotid angiography with the Matas maneuver showing collateral flow to the right anterior and middle cerebral arteries via the anterior communicating artery and retrograde filling to the CCF. The right anterior and middle cerebral arteries are not sufficiently opaque compared with the contralateral counterparts due to arterial steal to the CCF. $(\mathbf{F}, \mathbf{G})$ Frontal $(\mathbf{F})$ and lateral $(\mathbf{G})$ views on left vertebral angiography with the Allcock maneuver showing collateral flow via the posterior communicating artery. The location and size of the fistula were more clearly visualized. 3D-TOF: 3D time of flight; CCF: carotid cavernous fistula; CS: cavernous sinus; ICA: internal carotid artery; MIP: maximum intensity projection; SOV: superior ophthalmic vein

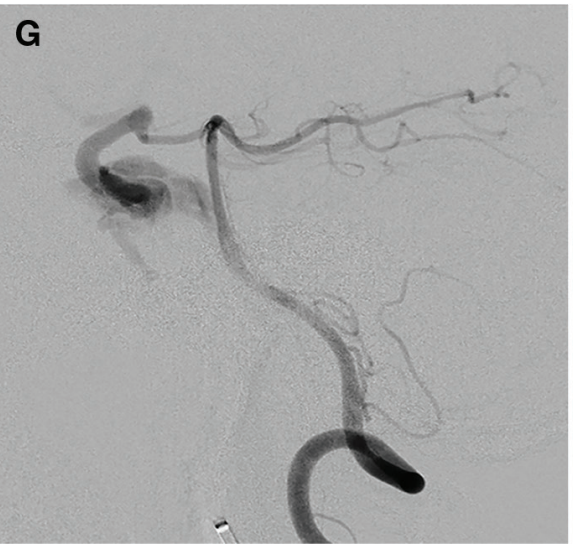



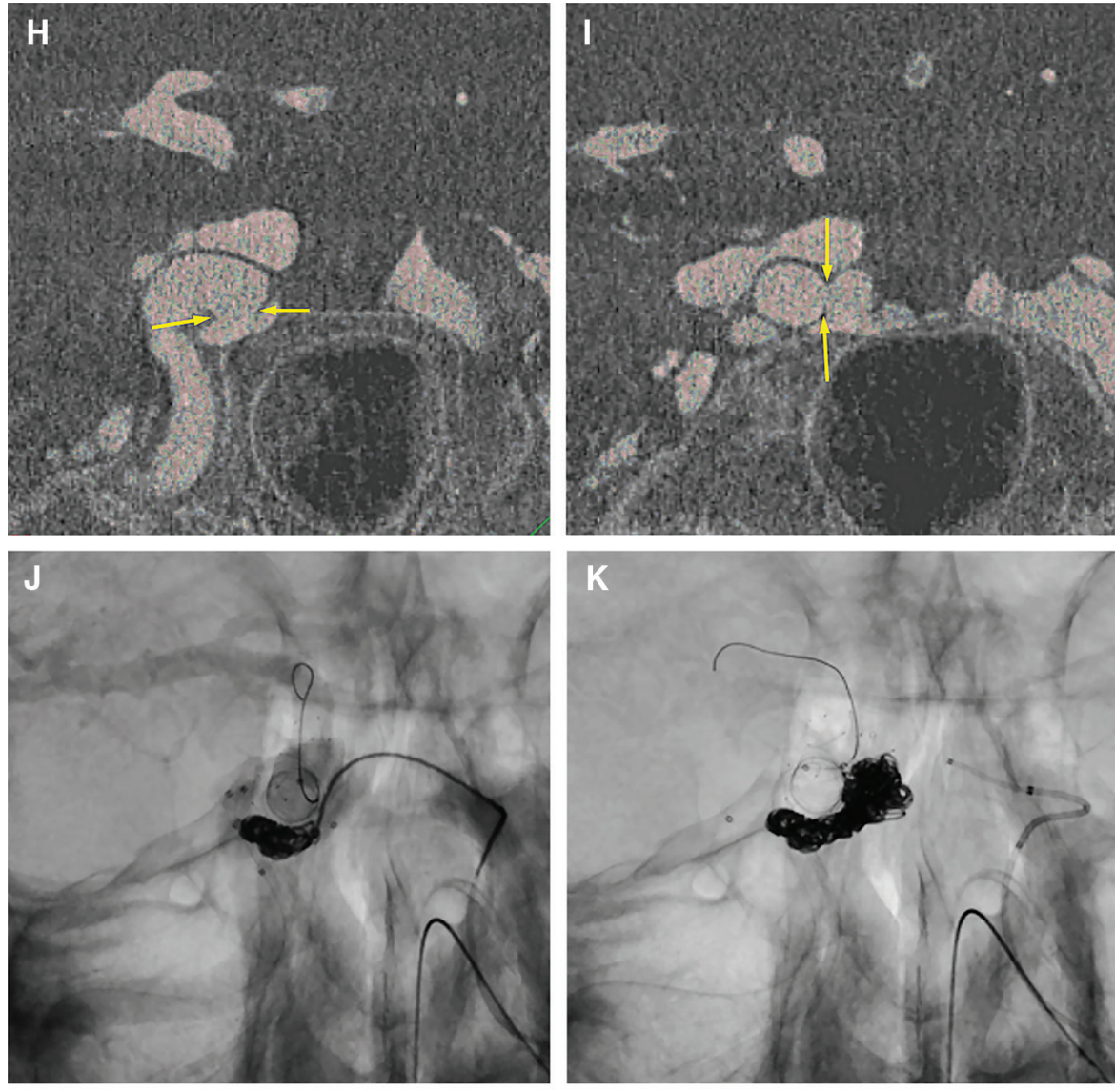

Fig. $1(\mathrm{H}, \mathrm{I})$ Coronal reconstructed 3D rotational angiography images of the right ICA $(\mathbf{H}, \mathbf{I}$; from the dorsal to ventral side) clearly showing two fistulas (yellow arrows) located at the inner wall and inferior wall of the horizontal cavernous portion of the right ICA. The CCF was treated by selective coil embolization using balloon- and stent-assisted techniques. Via both transarterial and transvenous approaches, two microcatheters were transvenously introduced into a fistulous pouch located inferiorly to the ICA, and another microcatheter was introduced into the same pouch transarterially. Coils were first placed into the fistulous pouch under the balloon- assisted technique. (J) Fluoroscopic image from the frontal view during balloon-assisted coil embolization showing that coils were introduced via three microcatheters (one via the right IPS, another via the left IPS, and the other via the ICA) placed in the same fistulous pouch located inferiorly to the ICA. (K) Fluoroscopic image from the frontal view after embolization. As there were two fistulas and the inferior one was too large to sufficiently pack the coils densely, selective coil embolization was continued using the stent-assisted technique. (L) Right internal carotid angiography after embolization showing complete occlusion of the CCF while preserving the right ICA. CCF: carotid cavernous fistula; ICA: internal carotid artery; IPS: inferior petrosal sinus; (The color version is available online.)

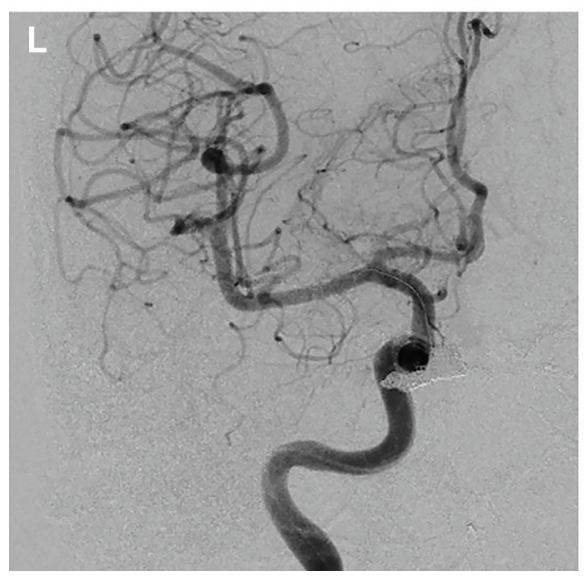

1974. ${ }^{11)}$ Transarterial balloon embolization of direct CCFs became the standard method in the 1980s. Coil embolization of the CS using detachable coils has recently become the standard management for direct CCFs due to the unavailability of detachable balloons in some countries, including Japan. Following recent advances in device technology for endovascular treatment, such as liquid embolic materials, covered stents, and flow-diverting stents, additional or alternative techniques have been applied for the treatment of direct CCFs.

\section{Detachable balloon and coils}

The advantage of balloon occlusion of a CCF is the ability to occlude the fistula rapidly with preservation of the ICA. The reported occlusion rates of fistulas with preservation of the ICA are high at around $75 \%-88 \% .{ }^{9,13,14)}$ However, technical difficulties, including insufficient embolization due to early balloon detachment, deflation, or rupture, are noted. Excessive inflation of the balloon can compress the cranial nerves and may induce cranial nerve palsy. During follow-up, distal migration of detachable balloons may 

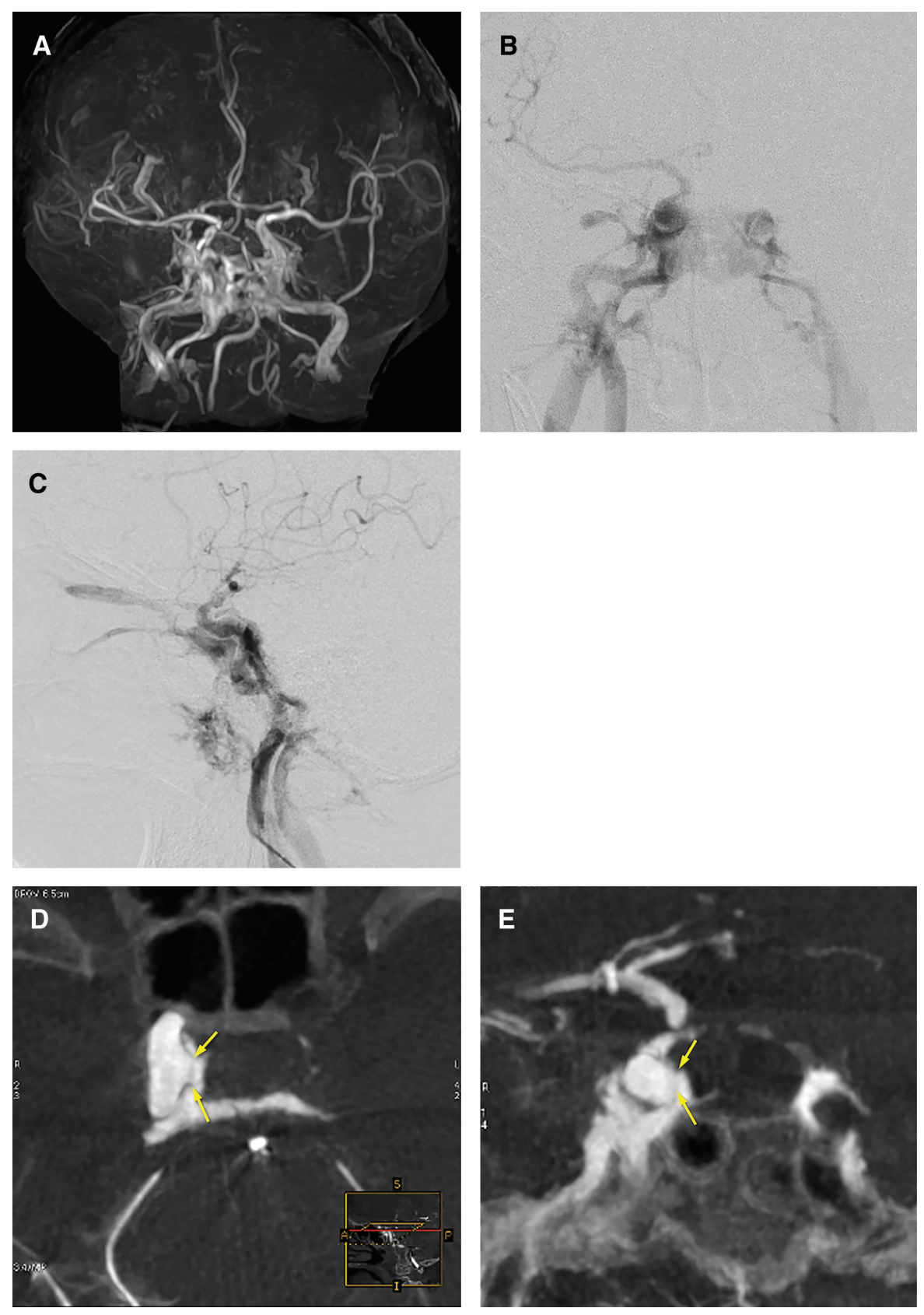

Fig. 2 A 91-year-old female with traumatic direct CCFs. (A) Coronal view on 3D-TOF MRA showing the high signal intensity of the bilateral $\mathrm{CS}$ and the right SOV. (B, C) Frontal (B) and lateral (C) views on right internal carotid angiography showing the high-flow CCF at the right cavernous portion. The direct $\mathrm{CCF}$ drained into the right CS to the contralateral CS, the bilateral inferior petrosal sinus, the bilateral pterygoid plexus, and the bilateral SOVs. (D, E) Axial (D) and coronal $(E)$ reconstructed $3 D$ angiography images of the right ICA clearly depicting the location of the fistula (yellow arrows) and fistulous venous pouch medially to the ICA. The direct CCF was treated by balloon-assisted coil embolization. A micro balloon catheter was placed crossing over the fistula in the cavernous portion of the right ICA and a microcatheter was advanced transarterially into the fistula via the orifice. Via the transvenous approach, two microcatheters were advanced into the same fistulous pouch via the right inferior petrosal sinus. 3D-TOF: 3D time of flight; CCF: carotid cavernous fistula; CS: cavernous sinus; ICA: internal carotid artery; SOV: superior ophthalmic vein; (The color version is available online.) occur, which causes recurrent CCFand aggravation of symptoms. Recurrent CCF with blockage of the main drainage route (usually the inferior petrosal sinus) by the migrated balloon increases the cerebral venous pressure and may induce serious complications such as cerebral hemorrhage. Moreover, delayed cavernous ICA pseudoaneurysms related to ICA wall failure following deflation of the balloon are frequently associated with balloon-occluded direct CCFs in 30\%-91\%. ${ }^{15,16)}$ These pseudoaneurysms may progress and cause the cranial nerve palsy or recurrent CCF.
In previous reports, the postoperative ICA patency rates ranged from 59 to $88 \% .^{14,15)}$ However, more recently, the application of additional endovascular modalities, such as detachable coils and stent application, increased the number of available options.

Coil embolization for direct CCFs became popular after FDA approval of the Guglielmi detachable coil (GDC). Transarterial and/or transvenous CS packing (Fig. 3), or selective embolization of the fistulous pouch with coils (Figs. 1 and 2), is an alternate treatment option for direct CCFs. 

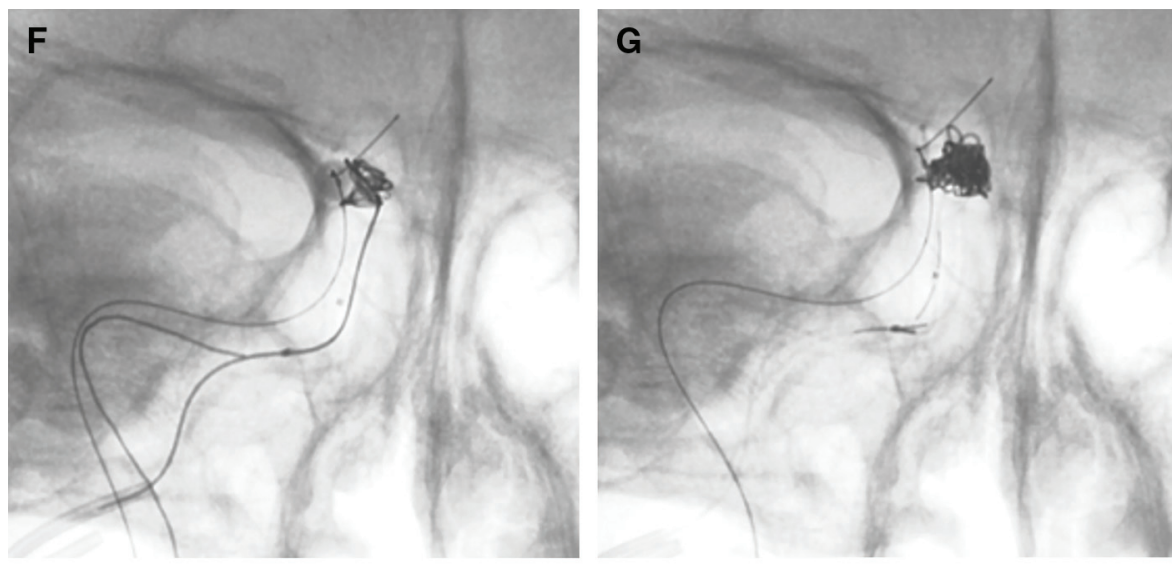

Fig. 2 (F) Fluoroscopic image showing three microcoils from three different microcatheters that were deployed into the fistula compartment under balloon inflation. (G) The fistulous pouch was packed with nine coils. $(\mathbf{H})$ Right internal carotid angiography after selective embolization showing complete occlusion of the CCF. CCF: carotid cavernous fistula;

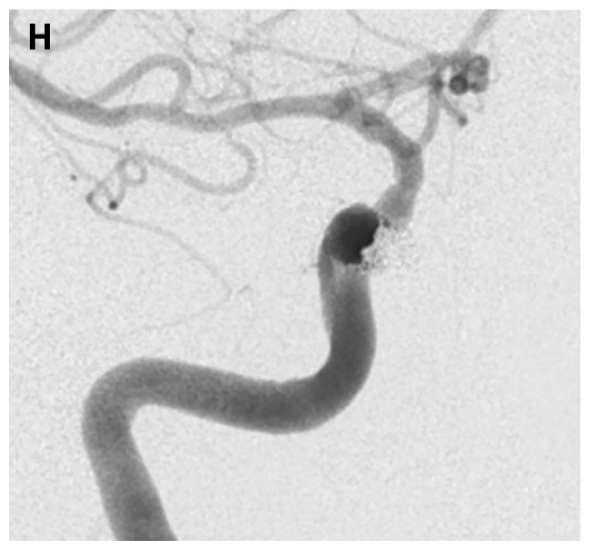

The advantageous aspects of coil application are their easy retrievability, ability to be repositioned, and better control. As detachable coils can be delivered through a smaller sized microcatheter, the microcatheter can be more easily inserted into the CS via a small tear that prevents passage of the balloon. This method can be applied for the majority of direct CCFs. However, there is a potential risk of protrusion or migration of coils into the parent artery when large/multiple tears are present. Adjunctive techniques of balloon-assisted or stent-assisted techniques to coil embolization were developed to overcome these difficulties. Balloon-assisted techniques are usually applied with transarterial and/or transvenous coil embolization in the majority of cases.

Stent-assisted coil embolization enables reconstruction of the damaged ICA with obliteration of the fistula (Fig. 1). Therefore, it is preferable in cases of large fistulas. There are only a few reports of stent-assisted coil embolization. Fanny E. Morón et al. ${ }^{17)}$ reported six cases of direct CCFs that were treated by stent-assisted coil embolization. Three cases were treated via the transvenous approach and the other three via were the transarterial approach. Direct $\mathrm{CCFs}$ were completely occluded without complication in all six cases. Administration of dual antiplatelet drugs before treatment is required to prevent acute stent thrombosis, but antiplatelet therapy may reduce the occlusive effects of the coil mass on direct CCFs.

Regarding approach routes to the CS and fistulous pouch (compartment), there are two approach routes, including the transarterial approach via the fistula and the transvenous approach via the inferior petrosal sinus. The transarterial approach is commonly used for detachable balloon embolization of direct CCFs. However, it is often difficult to navigate a detachable balloon via a small fistula. In 1988, Halbach et al. ${ }^{18)}$ reported that they treated 14 of 165 direct CCFs using the transvenous approach. ${ }^{18)}$ Soon after this, transvenous embolization became widely used for the treatment of CCFs as a solo technique or in combination with transarterial embolization, particularly using coils. The transvenous approach has a lower risk of thromboembolic complications as intra-arterial maneuvers are avoided. Especially in cases with vessel fragility, such as EhlersDanlos syndrome, the transvenous approach should be used to avoid arterial injury such as dissection along the catheterized arteries. ${ }^{5)}$ The CS connects to numerous veins, including superficial and deep middle cerebral 
veins, cerebellar-pontine veins, orbital and ocular veins, and pterygo/pharyngeal veins. Occlusion of an inadequate portion of the CS or venous out-flow of CCFs redirects outflow to the cerebral or ocular veins may aggravate ocular symptoms or cause intracerebral hemorrhage during or after transvenous embolization with sinus packing. Therefore, careful attention should be paid to the embolization site during transvenous embolization. Cranial nerve injury by compression from the coil mass is another potential drawback of sinus packing with coils. Cranial nerve palsy after sinus packing develops in approximately $39 \%-44 \%$ of cases. ${ }^{19,20)}$ Most cases are transit but it can become permanent (Fig. 3). Selective embolization of the fistulous pouch or compartment can occlude fistulas without aggravation of the drainage pattern or mass effect when the fistulous pouch or fistulous compartment of the CS is restricted (Fig. 2).

\section{Additional/alternative devices}

The utility of liquid embolic materials, such as histoacryl glue (N-butyl-2 cyanoacrylate n-BCA) and Onyx (EVOH: ethylene vinyl alcohol), for direct CCFs was recently reported by several authors. These materials were developed for the embolization of arteriovenous malformations. Luo et al. reviewed 176 patients with traumatic direct CCFs treated at their institution between 1992 and 2004. Among them, 18 had CCFs that were unable to be obliterated using balloon or coils and were treated by n-BCA. ${ }^{21)}$ According to their report, complete fistula occlusion with preservation of the ICA was achieved immediately after injection of n-BCA in 16 patients. In the remaining two, small residual shunt flow was noted on postembolization angiography, which spontaneously thrombosed at follow-up angiography 1 week later. Among the 16 patients with immediate occlusion after initial embolization, fatal subarachnoid hemorrhage and massive epistaxis developed in one each. Furthermore, other adverse events, including asymptomatic migration of n-BCA, ICA pseudoaneurysm, and temporary impairment of cranial nerve function, were observed in four, five, and three patients, respectively. More recently, Ohlsson et al. ${ }^{22)}$ reported their treatment results of direct CCFs using 91\% n-BCA-lipiodol and tantalum powder mixture alone in nine patients. Four patients underwent embolization of CCFs without occlusion of the parent artery and five patients with a large fistula were treated by embolization while sacrificing the parent artery. All nine CCFs were successfully obliterated without recurrence. In their series, there were no serious complications. One case each of groin hematoma and transient 6th nerve palsy was observed. Although they did not observe migration of the n-BCA mixture into the ICA, sufficient skill and experience are required to achieve complete occlusion of high-flow fistulas without unintentional glue reflux or distal migration.

Onyx has recently become the standard embolic material for transarterial and transvenous embolization of dural arteriovenous fistulas and brain arteriovenous malformations. ${ }^{23,24)}$ Several authors previously reported the use of Onyx adjunctive to coil embolization or Onyx alone for the embolization of direct CCFs.

High occlusion rates were reported $(90 \%-100 \%)$ and no recurrence during 6- to 12-month follow-up periods were described. Although no complications or low complication rates were described in a few reports, Du et al. ${ }^{25}$ ) reported complications related to Onyx embolization, including ICA occlusion in 5\% and new symptoms of oculomotor nerve damage in $23.7 \%$. One complication of cast embolization in the middle cerebral artery was also reported in another study. Furthermore, asystole and bradycardia due to trigeminal cardiac reflux stimulated by DMSO may develop. Trigeminal cardiac reflux during transvenous Onyx embolization of CS dural arteriovenous fistulas may develop in up to $18 \%$ of cases.

Placement of a covered stent or flow-diverting stent is a novel method to treat CCFs via their closure with simultaneous reconstruction of the injured ICA.

The use of covered stents for the treatment of direct CCFs has been reported sporadically over the last 18 years. In 2002, Kocer et al. reported the first case of traumatic CCF secondary to a pituitary surgery treated using a coronary stent graft. Gomez et al. ${ }^{26}$ ) reported seven cases in 2007 treated by coronary stent grafts and immediate occlusion of the fistula with ICA preservation was achieved in all. There were no immediate causes of mortality or morbidity, but occlusion of the ICA during was noted at 1-month follow-up. Gercken et al. ${ }^{27)}$ reported intrastent stenosis and stent thrombosis after placement of a stent graft in coronary or bypass artery in $31.6 \%$ and $5.7 \%$, respectively. Therefore, there is a potential risk of occlusion of the covered stent or ICA during long-term follow-up. On the other hand, recurrent $\mathrm{CCF}$ after closure of a direct $\mathrm{CCF}$ due to endoleak was also reported. Another drawback of the stent graft system is its rigid properties due to the lack of commercialized devices for intracranial vessels, which make maneuvering difficult, and cause stress to the injured ICA during navigation and deployment of stent graft. Complications of dissection of the ICA and early deployment of the stent graft during placement have been reported. ${ }^{27,28)}$ 

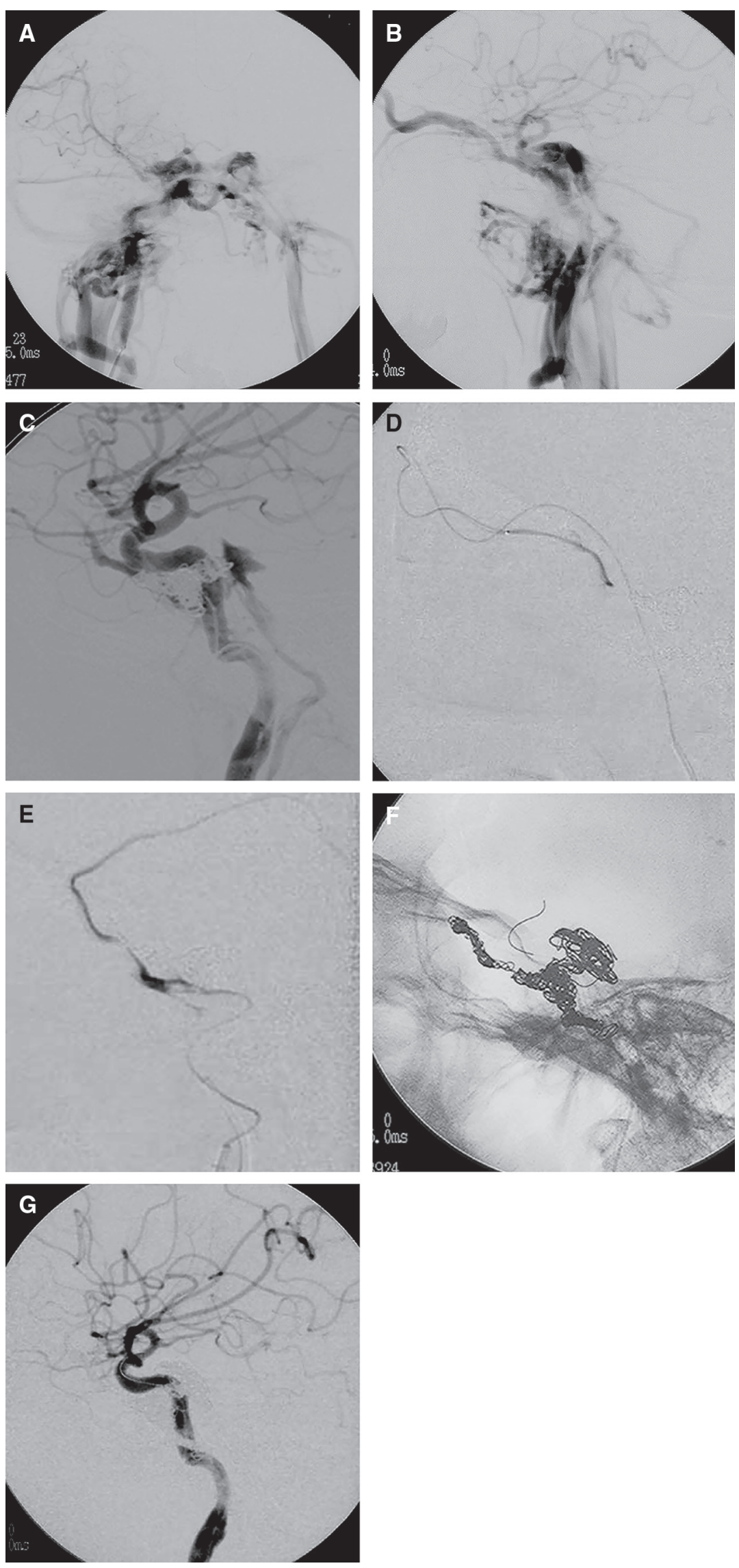

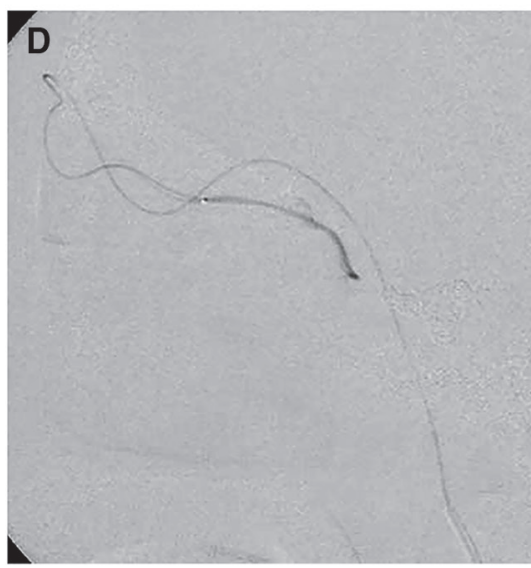

Fig. 3 A case of traumatic CCF with delayed abducens nerve palsy that developed 5 years after embolization by CS packing with coils. (A, B) Frontal (A) and lateral (B) views on right internal carotid angiography showing a high-flow direct CCF draining into the right SOV, right superior petrosal sinus, contralateral CS via the inter-CS, bilateral IPS, and bilateral pterygoid plexus. A microcatheter was advanced into the CS via the fistula and the posterior compartment of the CS was embolized using coils. (C) Lateral view on right internal carotid angiography after transarterial embolization showing a small residual CCF draining into the right inferior petrosal sinus and the right SOV. Right abducens nerve palsy developed a few days after embolization. As the CCF remained on follow-up angiography a few weeks later, repeated embolization was performed via both transvenous and transarterial approaches. (D) Selective angiography of the CS via the catheter introduced via the right superficial temporal vein, angular vein, and the SOV. The anterior part of the CS and the SOV was embolized using coils. Then, the microcatheter was advanced via the right inferior petrosal sinus into the right superficial middle cerebral vein. (E) Selective angiography of the right superficial middle cerebral vein showing cortical venous reflux. The proximal portion of the superficial middle cerebral vein and the lateral compartment of the CS were embolized using coils. Then, a microcatheter was advanced from the ICA via the tear into the posterior compartment of the CS, and the compartment was embolized using coils under the balloon-assisted technique. (F) Lateral views on fluoroscopy at the end of the embolization pro-

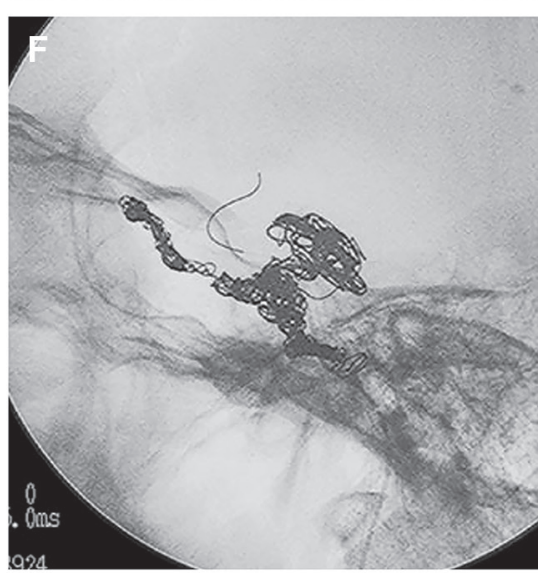
cedures showing the coil mass in the entire CS. (G) Lateral view on right internal carotid angiography showing complete occlusion of the CCF. The patient's symptoms completely resolved within 3 months after embolization. However, right abducens nerve palsy recurred 5 years after embolization. Repeated angiography and head MRI demonstrated no recurrence of the CCF or other abnormal findings. The recurrent abducens nerve palsy became a permanent deficit. 3D-TOF: 3D time of flight; CCF: carotid cavernous fistula; CS: cavernous sinus; ICA: internal carotid artery; IPS: inferior petrosal sinus; SOV: superior ophthalmic vein 
Although direct CCFs are well known as a complication of flow-diverting stent placement in the treatment of cavernous ICA aneurysms, few reports have demonstrated the use of flow-diverting stents for treating direct CCFs. Amuluru et al. reported a case of direct CCF that developed during flow-diverting stent placement for an unruptured cavernous ICA aneurysm. They treated the direct CCF using a second flow-diverting stent without additional embolization. ${ }^{29)}$ Although favorable outcomes with CCF occlusion and clinical improvement were noted in these limited case reports, ${ }^{30}$ ) the true efficacy of flow-diverting stents is unclear.

Furthermore, both reconstructive techniques using a stent graft and flow-diverter require the long-term use of dual antiplatelet drugs after placement to prevent intrastent thrombosis. Therefore, the use of these reconstructive techniques for direct CCFs is limited to selected cases.

\section{Parent artery occlusion}

Parent artery occlusion (PAO) may be required when endovascular occlusion of a direct CCF with preservation of the ICA is not feasible due to extensive wall damage of the injured ICA or emergency cases with active nasopharyngeal bleeding. Although sacrifice of the ICA is usually possible in cases of long-standing CCF without distal flow of the ipsilateral ICA to the cerebral parenchyma beyond the CCF, balloon test occlusion is generally recommended to ensure distal perfusion via collateral circulation from the anterior and posterior communicating arteries, and to evaluate the size and exact location of the fistula before PAO. PAO can be performed using many endovascular techniques, such as coil, balloon, and vascular plug embolization, and it is more reliable for occlusion of direct CCFs. Dai et al. ${ }^{31)}$ reported 126 cases of direct CCFs, including 52 treated by PAO. Among these patients, Horner's syndrome developed in three patients. No ischemic stroke was noted in the PAO group or ICA preserved group. During follow-up, CCFs recurred in nine patients $(12.2 \%)$ in the ICA preserved group, but in no patients in the PAO group. Although good treatment results were reported by Dai et al., the PAO procedure has a potential risk of cerebral infarction due to both hypoperfusion and embolism from stagnated blood at the stump distal to the occlusion site. Even among patients tolerant to balloon test occlusion, the incidence of neurological deficits after PAO is $3 \%-7 \%{ }^{32}$ )

\section{Summary}

There are various treatment options for direct CCFs. The basic technique is balloon-assisted coil embolization of the
$\mathrm{CS}$, which can be applied in the majority of cases. Selection of additional/alterative techniques and devices depends on the anatomy and hemodynamics of each CCF, and the skill and experience of individual operators.

\section{Disclosure Statement}

The authors declare that they have no conflict of interest.

\section{References}

1) Lylyk P, Viñuela F, Campos J, et al: Diagnosis and endovascular therapy of vascular lesions in the cavernous sinus. Acta Radiol Suppl 1986; 369: 584-585.

2) Fleishman JA, Garfinkel RA, Beck RW, et al: Advances in the treatment of carotid cavernous fistulas. Int Ophthalmol Clin 1986; 26: 301-311.

3) Barrow DL, Spector RH, Braun IF, et al: Classification and treatment of spontaneous carotid-cavernous sinus fistulas. J Neurosurg 1985; 62: 248-256.

4) Ringer AJ, Salud L, Tomsick TA: Carotid cavernous fistulas: anatomy, classification, and treatment. Neurosurg Clin N Am 2005; 16: 279-295.

5) Van Overmeire O, De Keukeleire K, Van Langenhove P, et al: Carotid-cavernous fistula in ehlers-danlos syndrome by pure transvenous approach. Interv Neuroradiol 2006; 12: $45-51$.

6) Roy AK, Grossberg JA, Osbun JW, et al: Carotid cavernous fistula after pipeline placement: a single-center experience and review of the literature. J Neurointerv Surg 2017; 9: 152-158.

7) Lin LM, Colby GP, Jiang B, et al: Transvenous approach for the treatment of direct carotid cavernous fistula following Pipeline embolization of cavernous carotid aneurysm: a report of two cases and review of the literature. BMJ Case Reports 2014; 2014: bcr2014011235.

8) Matsuda T, Satoh K, Hanaoka M, et al: Evaluation of stenting for intracranial internal carotid artery stenosis using flow reversal. J Neuroendvasc 2018; 12: 213-218.

9) Debrun G, Lacour P, Vinuela F, et al: Treatment of 54 traumatic carotid-cavernous fistulas. J Neurosurg 1981; 55: 678-692.

10) Chi CT, Nguyen D, Duc VT, et al: Direct traumatic carotid cavernous fistula: angiographic classification and treatment strategies. Study of 172 cases. Interv Neuroradiol 2014; 20: 461-475.

11) Serbinenko FA: Balloon catheterization and occlusion of major cerebral vessels. J Neurosurg 1974; 41: 125-145.

12) Travers B: A case of aneurism by anastomosis in the orbit, cured by the ligature of the common carotid artery. Med Chir Trans 1811; 2: 1-420.1. 
Ide S, et al.

13) Lu X, Hussain M, Ni L, et al: A comparison of different transarterial embolization techniques for direct carotid cavernous fistulas: a single center experience in 32 patients. $J$ Vasc Interv Neurol 2014; 7: 35-47.

14) Lewis AI, Tomsick TA, Tew JM, et al: Management of 100 consecutive direct carotid-cavernous fistulas: results of treatment with detachable balloons. Neurosurgery 1995; 36: 239-244.

15) Luo CB, Teng MM, Yen DH, et al: Endovascular embolization of recurrent traumatic carotid-cavernous fistulas managed previously with detachable balloons. J Trauma 2004; 56: 1214-1220.

16) Marques MC, Caldas JG, Nalli DR, et al: Follow-up of endovascular treatment of direct carotid-cavernous fistulas. Neuroradiology 2010; 52: 1127-1133.

17) Morón FE, Klucznik RP, Mawad ME, et al: Endovascular treatment of high-flow carotid cavernous fistulas by stent-assisted coil placement. AJNR Am J Neuroradiol 2005; 26: 1399-1404.

18) Halbach VV, Hieshima GB, Higashida RT, et al: Carotid cavernous fistulae: indications for urgent treatment. AJR Am J Roentgenol 1987; 149: 587-593.

19) Bink A, Goller K, Lüchtenberg M, et al: Long-term outcome after coil embolization of cavernous sinus arteriovenous fistulas. AJNR Am J Neuroradiol 2010, 31: 1216-1221.

20) Nishino $K$, Ito $Y$, Hasegawa $H$, et al: Cranial nerve palsy following transvenous embolization for a cavernous sinus dural arteriovenous fistula: association with the volume and location of detachable coils. J Neurosurg 2008; 109: 208-214.

21) Luo C, Teng M, Chang F-C, et al: Transarterial balloonassisted n-butyl-2-cyanoacrylate embolization of direct carotid cavernous fistulas. AJNR Am J Neuroradiol 2006, 27: $1535-1540$.

22) Ohlsson M, Consoli A, Rodesch G, et al: Endovascular treatment of carotico-cavernous fistulas with acrylic glue: a series of nine cases. Neuroradiology 2016, 58: 1181-1188.
23) $\mathrm{Hu} \mathrm{YC}$, Newman CB, Dashti SR, et al: Cranial dural arteriovenous fistula: transarterial Onyx embolization experience and technical nuances. J Neurointerv Surg 2011; 3: $5-13$.

24) Hiramatsu M, Sugiu K, Hishikawa T, et al: Results of 1940 embolizations for dural arteriovenous fistulas: Japanese Registry of Neuroendovascular Therapy (JR-NET3). $J$ Neurosurg June 28, 2019 [Epub ahead of print].

25) Du B, Zhang M, Wang Y, et al: A retrospective analysis of 38 carotid cavernous fistula patients treated with balloon-assisted endovascular fistula embolization through simultaneous transarterial and transvenous approaches. Int J Clin Exp Med 2016; 9: 19399-19407.

26) Gomez F, Escobar W, Gomez AM, et al: Treatment of carotid cavernous fistulas using covered stents: midterm results in seven patients. AJNR Am J Neuroradiol 2007; 28: 1762-1768.

27) Gercken U, Lansky AJ, Buellesfeld L, et al: Results of the Jostent coronary stent graft implantation in various clinical settings: procedural and follow-up results. Catheter Cardiovasc Interv 2002; 56: 353-360.

28) Baek JW, Kim ST, Lee YS, et al: Recurrent carotid cavernous fistula originating from a giant cerebral aneurysm after placement of a covered stent. J Cerebrovasc Endovasc Neurosurg 2016; 18: 306-314.

29) Amuluru K, Al-Mufti F, Gandhi CD, et al: Direct carotid-cavernous fistula: a complication of, and treatment with, flow diversion. Interv Neuroradiol 2016; 22: 569-576.

30) Patel PD, Chalouhi N, Atallah E, et al: Off-label uses of the Pipeline embolization device: a review of the literature. Neurosurg Focus. 2017; 42: E4.

31) Dai RQ, Bai WX, Gao BL, et al: Internal carotid artery occlusion may affect long-term quality of life in patients with high-flow carotid cavernous fistulas. Interv Neuroradiol 2020; 26: 83-89.

32) American Society of Interventional and Therapeutic Neuroradiology: Carotid artery balloon test occlusion. AJNR Am J Neuroradiol 2001; 22: S8-S9. 